\title{
Análisis coste-beneficio y coste-efectividad, de las nuevas alternativas de inmunización surgidas en el mercado para la prevención de la leishmaniosis canina en las Fuerzas Armadas
}

\author{
Salinas Granell MB. ${ }^{1}$, Tabanera de Lucio A. ${ }^{2}$ \\ Sanid. mil. 2013; 69 (4): 249-256; ISSN: 1887-8571
}

\begin{abstract}
RESUMEN
Antecedentes y objetivos: La incorporación de estudios epidemiológicos y métodos de análisis de costes suponen herramientas de primer orden dentro de la Sanidad Militar. Permiten optimizar los recursos y estructuras disponibles, manteniendo así la eficacia y efectividad en la lucha frente a las enfermedades, una de ellas la Leishmaniosis. La epidemiología de campo, se nos presenta como una útil herramienta para alcanzar nuestro objetivo sanitario estratégico que es el de controlar esta enfermedad. La leishmaniosis canina es una de las parasitosis de mayor repercusión en las Fuerzas Armadas tanto desde el punto de vista de salud pública como de operatividad de nuestros perros de trabajo militar. El trabajo tiene como objetivo comparar el coste-beneficio (C-B) de dos tipos de vacunas con los costes que supondría la externalización de los servicios. Para ello se analiza el coste-efectividad (C-E), que supondría ampliar el actual programa de vacunación, con la incorporación de la nueva vacuna frente a LI (CaniLeish $\left.{ }^{\circledR}\right)$, y analizar el costeefectividad (C-E) que supondría ampliar el actual programa preventivo antivectorial con la adquisición de un nuevo medicamento preventivo frente a LI (Leisguard ${ }^{\circledR}$ ). Material y Método: Se ha estimado mediante el análisis coste-beneficio y coste-efectividad, la eficiencia que supondría, teniendo en cuenta la seroprevalencia actual, el ampliar el existente programa preventivo con la adquisición de las nuevas alternativas de inmunización (vacunación y uso de domperidona) surgidas en el mercado frente a la leishmaniosis canina. Requiriéndose una valoración monetaria apropiada de los efectos sobre la salud de las alternativas evaluadas. Resultados: Se obtuvo que los costes globales de ambas alternativas son superiores al coste del tratamiento de la enfermedad, con relaciones coste-beneficio y coste-efectividad negativas (en términos de coste por mantenimiento de operatividad del efectivo canino), es decir, no se produce ahorro neto. Conclusiones: Derivado de los resultados obtenidos en estas técnicas analíticas concluimos que, el uso de recursos alternativos no reporta eficiencia frente a las actuaciones actuales. En este caso, cualquier medida preventiva nueva debe considerarse a medio/corto plazo, y siempre en función de los cambios epidemiológicos de la enfermedad en cuestión.
\end{abstract}

PALABRAS CLAVE: Coste, Vacunación, Inmunidad celular, Leishmaniosis canina, Fuerzas Armadas.

Cost-benefit and cost-effectiveness analysis of the new immunization alternatives for the prevention of canine leishmaniasis in the Armed Forces. SUMMARY: Antecedents and objectives: Epidemiological studies and cost analysis methods are first-rate tools in the Medical Service. They allow to optimize the available resources and structures, maintaining efficacy and effectiveness in the fight against diseases, one of them the leishmaniasis. Field epidemiology is a useful tool to reach our health strategic goal of controlling this disease. Canine leishmaniasis is one the most important parasitic diseases in the Armed Forces, both from the point of view of public health and the operational capacity of our military working dogs. This study intends to compare the cost-benefit (C-B) of two types of vaccines with the costs of outsourcing the services. In order to do this the cost- effectiveness (C-E) of expanding the current immunization calendar, including a new vaccine against LI (CaniLeish $\left.{ }^{\circledR}\right)$ and the cost-effectiveness $(\mathrm{C}-\mathrm{E})$ of expanding the current vector control program with the acquisition of a new preventive drug against LI (Leisguard ${ }^{\circledR}$ ), are evaluated. Material and Method: the efficiency of expanding the existing preventive program with the new immunization alternatives (vaccination and the use of domperidone) appeared in the market against canine leishmaniasis, has been studied through a cost-benefit and cost-effectiveness evaluation, taking into account the current seroprevalence. An adequate monetary valuation of the impact on health of the studied alternatives is required. Results: the overall costs of both alternatives are higher than the cost of the treatment of the disease, with negative cost-benefit and cost-effectiveness relationships (in terms of cost per operational maintenance of the dogs), that is, there are no net savings. Conclusions: from the results obtained with these analytical techniques we conclude that the alternative resources are not more efficient that the current activities. In this case, any new preventive measure must be considered in the medium / short term and always bearing in mind the epidemiological changes of the disease concerned.

KEY WORDS: Cost, Vaccination, Cellular immunity, Canine leishmaniasis, Armed Forces.

${ }^{1}$ Cap. Veterinario. Escuela Militar de Sanidad. Madrid. España.

${ }^{2}$ Cte. Veterinario. Centro Militar de Veterinaria de la Defensa. Servicio de Microbiología, Higiene y Sanidad Ambiental. Madrid. España.

Dirección para correspondencia: Mireya Salinas Granell. Escuela Militar de Sanidad. C/Camino de los Ingenieros nº 6.28047 Madrid. email: msalgra@oc.mde.es

Recibido: 16 de mayo de 2013

Aceptado: 7 de septiembre de 2013

\section{INTRODUCCIÓN}

La leishmaniosis, enfermedad causada por protozoos del género Leishmania, afecta fundamentalmente al hombre y al perro, y engloba un amplio espectro de procesos patológicos que pueden ir desde cuadros cutáneos autorresolutivos a procesos 


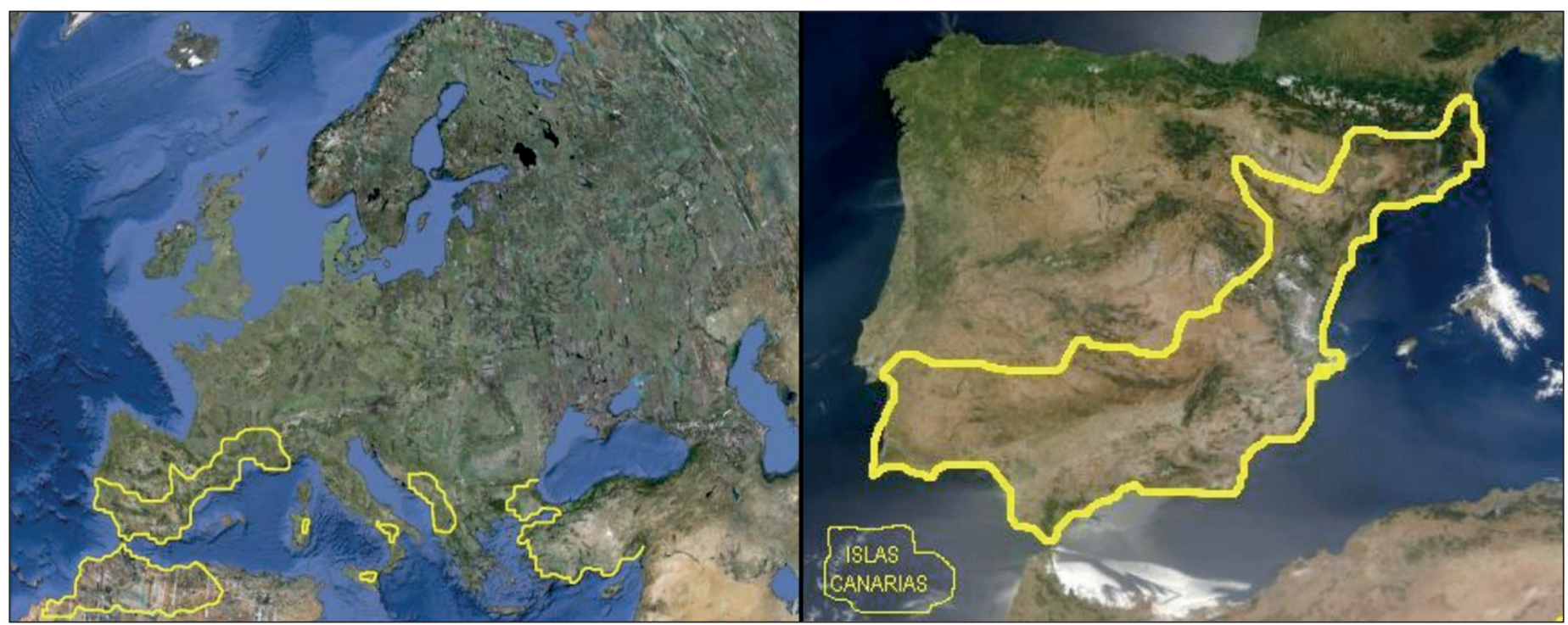

Figura 1. Distribución geográfica de la Leishmaniosis visceral.

viscerales de terminación fatal. Es transmitida por insectos flebótomos y se caracteriza por la parasitación de las células del sistema fagocitario del hospedador ${ }^{1}$.

Es una parasitosis canina con una doble repercusión: por un lado en salud pública, dado el carácter zoonótico de la enfermedad, y por otro en veterinaria, en la que por su notable incidencia, dificultad del diagnóstico precoz y relativa falta de eficacia del tratamiento, constituye una enfermedad altamente problemática.

En Europa se presentan de forma endémica dos formas de leishmaniosis: cutánea y visceral, aunque con baja prevalencia, ambas producidas por el parásito Leishmania infantum (LI).

En España, la prevalencia media de la parasitación puede situarse entre el 5-10\% de la población canina, llegando alcanzar el $25 \%$ en algunas zonas de la cuenca mediterránea y el 50\% en el archipiélago canario (Figura 1) ${ }^{1}$.

Los flebótomos, y más específicamente Phebotomus perniciosus y Phebotomus ariasi, son los únicos vectores biológicos demostrados de Leishmania infantum ${ }^{2}$.

Pese a esta información, los datos que manejamos en la sección de análisis clínicos, parasitología y epidemiología del Centro Militar de Veterinaria de la Defensa (CEMILVETDEF), sobre las zoonosis especificadas en la Instrucción Técnico Sanitaria (ITS) $03 / 2007^{3}$, nos muestran que los efectivos caninos de nuestras Fuerzas Armadas (FFAA) padecen una prevalencia a LI muy distinta a la que padecen los «perros civiles».

Así, nos encontramos que de 923 muestras analizadas ${ }^{4}$ mediante inmunofluorescencia (IFI) frente a LI, se obtuvieron positivas con una titulación $1: 200(n=50 ; 5,41 \%)$, dudosas con una titulación $1: 100(n=36 ; 4,02 \%)$ y negativas con una titulación $1: 50(n=837 ; 90,6 \%)$.

Cabe destacar que la prevalencia de aparición de la enfermedad en el efectivo canino de las FFAA está decreciendo anualmente, siendo de $2,25 \%$ en el año 2011 .

En la actualidad existe un debate creciente sobre los recursos en la sanidad militar. Por tanto se debe plantear cómo deben ser manejados estos en la atención de los animales de interés militar.
Por una parte nos encontramos con que las necesidades de salud del animal en el perro de trabajo militar (PTM), son muy numerosas, y por otra, que los recursos financieros para salud son limitados. Esta disyuntiva que se nos plantea no tiene una solución fácil, máxime cuando se trata de una zoonosis.

Para realizar este trabajo hemos tenido en cuenta la definición que sobre la economía de la salud propugna Baly y colaboradores al afirmar que: «Es la ciencia que estudia la distribución de los escasos recursos disponibles para obtener el máximo rendimiento posible, medido a través de la mejoría en los indicadores de cantidad y calidad de la salud» ${ }^{5}$.

Nuestro fin principal es mantener sanos a nuestros perros de trabajo militar (PTM), para tratar de aumentar la operatividad disminuyendo las pérdidas que originarían enfermedades evitables, tanto si evolucionan con sintomatología aparente como si lo hacen de forma inaparente (subclínica), en ocasiones con escasa mortalidad, pero ocasionando elevadas pérdidas económicas como es el caso de la LI.

Hay que objetivar los gastos que consideramos fijos en un PTM, para que desempeñe su función y sea operativo. Gastos que lleva asumiendo Veterinaria Militar hasta la fecha con medios humanos y recursos materiales y que son los siguientes:

- Valor del animal en origen.

- Pruebas diagnósticas de compra.

- Programa de vacunación.

- Programa de desparasitación.

- Programa preventivo antivectorial.

- Programa diagnóstico de la Instrucción Técnica Sanitaria 03/2007.

- Programa de alimentación.

- Programa de atención clínica veterinaria.

- Programa de adiestramiento.

A estos gastos, tendríamos que añadir los nuevos programas preventivos a considerar:

- Vacuna contra la leishmaniosis canina, 2012. CaniLeish ${ }^{\circledR}$ (Virbac).

- Tratamiento de la enfermedad, 2012. Leisguard ${ }^{\circledR}$ (Esteve). 
Análisis coste-beneficio y coste-efectividad, de las nuevas alternativas de inmunización surgidas en el mercado para la ...

Debemos efectuar en este punto de la introducción, una somera descripción científica de los dos modelos a estudiar, con la finalidad de poder establecer un criterio ecuánime a la hora de la elección basado en la evidencia.

\section{CaniLeish $^{\circledR}$ (Virbac)}

Es la primera vacuna contra la leishmaniosis canina en Europa desarrollada por los laboratorios Virbac 6

La literatura científica recogida por el propio laboratorio dice: «Es una vacuna que estimula una respuesta inmunitaria celular apropiada y específica contra el parásito LI».

Lo que esta nueva vacuna induce contra la LI, es a una inmunidad mediada por células, sólida y específica, hacia este parásito intracelular. Este efecto lo consiguieron mediante el diseño de un medio de cultivo (patentado por Virbac) sin suero ni células, para reproducir el ciclo biológico completo de la LI sin utilizar animales de experimentación. Lo que obtuvieron en este medio de cultivo fueron proteínas que todas ellas fueron exclusivamente producidas por el parásito de LI, siendo denominadas proteínas secretadas excretadas (PSE).

Estas PSE son altamente inmunógenas para una respuesta inmunitaria apropiada contra LI y estimulan mejor la proliferación de linfocitos T que el extracto del parásito entero ${ }^{7}$.

Por otro lado esta vacuna incluye como adyuvante el QA-21, que es una saponina purificada componente de Quil-A, que es un adyuvante sinérgico que optimiza la respuesta inmunitaria celular pues estimula la respuesta Th-1 y la producción de linfocitos $\mathrm{T}$ citotóxicos, con un excelente perfil de seguridad general como ha quedado demostrado en la vacuna contra la leucemia felina (Leucogen ${ }^{\circledR}$ ).

Los ensayos de campo comunicados por los laboratorios Virbac, obtienen unos resultados del $92,7 \%$ de animales que no desarrollaron la parasitosis sintomática.

\section{Pauta de administración ${ }^{6}$}

Se recomienda en primer lugar la realización de un test de diagnóstico de la enfermedad. Si el test es negativo, puede iniciarse la primovacunación con tres dosis subcutáneas:

- La primera dosis a partir de los seis meses de edad.

- La segunda y tercera dosis se administra a intervalos de tres semanas.

- Revacunación anual.

\section{Leisguard ${ }^{\circledR}$ (Esteve)}

Leisguard ${ }^{\circledR}$ es un nuevo medicamento comercializado por los laboratorios Esteve, que tiene según el propio laboratorio como función terapéutica: «El tratamiento y la prevención de la leishmaniosis canina $»^{8}$, y se trata de una suspensión oral cuyo principio activo es la domperidona.

La domperidona es un derivado bencimidazólico que actúa a través del bloqueo específico de los receptores dopaminérgicos D2 a nivel periférico y cuya administración repetida en perros sanos induce a un incremento progresivo de la actividad fagocítica de las poblaciones de neutrófilos y monocitos en sangre periférica, lo cual deriva en una mayor resistencia de estas células frente a la infección experimental in vitro con amastigotes de Leishmania (datos no publicados, según autores) ${ }^{9}$.

Esta droga es ampliamente utilizada en humanos y perros como agente antiemético y gastrocinético, siendo ambas actividades debidas al bloqueo de los receptores dopaminérgicos D2 a nivel del centro del vómito integrado en el bulbo raquídeo y a nivel del tracto digestivo superior, respectivamente ${ }^{10,11}$; menos conocida es su actividad endocrina hiperprolactinémica derivada del bloqueo de los receptores dopaminérgicos D2 a nivel de la glándula pituitaria o hipófisis.

Este bloqueo conlleva la liberación aguda de un pico de prolactina acumulada en la hipófisis lo cual deriva en un pico transitorio de pocas horas de duración en los niveles sanguíneos de esta hormona ${ }^{12,13}$. Lo que consigue la domperidona es la estimulación del sistema inmunológico mediante picos de prolactina, para obtener una respuesta específica en caso de picadura de mosquito ${ }^{14-17}$.

Basándose en este fenómeno, justifican el uso de la domperidona para la prevención de la leishmaniosis canina, además de su uso terapéutico en perros enfermos ${ }^{18-19}$.

$\mathrm{Su}$ administración oral repetida en perro tiene un efecto estimulante sobre el sistema inmunitario tanto a nivel de la respuesta innata como de la adquirida, contribuyendo al establecimiento de una respuesta de tipo predominantemente celular (Th 1$)^{20}$.

\section{Pauta de administración ${ }^{8}$}

La pauta de administración de Leisguard ${ }^{\circledR}$ tanto en animales enfermos como en aquellos sanos en zonas de riesgo de contagio es de una vez al día durante 1 mes, repitiendo el tratamiento cada 4 meses.

Para su uso preventivo su administración debe realizarse de forma periódica a lo largo de todo el año de forma estratégica en función de la temporada de transmisión del parásito y la prevalencia de la enfermedad en una zona geográfica concreta9 9

\section{OBJETIVOS}

Basándonos en lo expuesto nos proponemos:

- Comparar el coste-beneficio (C-B) de ambos modelos con los costes que supondría la externalización de los servicios.

- Analizar el coste-efectividad (C-E) que supondría el ampliar el actual programa de vacunación con la incorporación de la nueva vacuna frente a LI (CaniLeish $\left.{ }^{\circledR}\right)$.

- Analizar el C-E que supondría ampliar el actual programa preventivo antivectorial con la adquisición de un nuevo medicamento preventivo frente a LI (Leisguard $\left.{ }^{\circledR}\right)$.

\section{MATERIAL Y MÉTODO}

Hemos llevado a cabo un estudio a partir de 1.200 sueros caninos, mediante IFI durante el año 2011, en la Sección de 
análisis clínicos, parasitología y epidemiología, perteneciente al Servicio de Microbiología, Higiene y Sanidad Ambiental del CEMILVETDEF. Sección que es la responsable y encargada de dar cumplimiento a la ITS 03/20073.

Se ha evaluado mediante los análisis C-B y C-E $\mathrm{E}^{21}$, la eficiencia de dos programas de inmunización preventivos de nueva aparición en el mercado, frente a la leishmaniosis canina; es decir, la utilización de la vacuna CaniLeish ${ }^{\circledR}$ de los laboratorios Virbac Salud Animal, o el uso del medicamento Leisguard ${ }^{\circledR}$ de los laboratorios Esteve.

Comparando los costes y beneficios de cada uno de ellos con la estrategia consistente en el tratamiento de los casos de leishmaniosis canina.

El C-E es por tanto, un coste efectividad incremental ${ }^{22-23}$ (No se ha realizado un estudio de sensibilidad por no haber considerado el IPC anual en el incremento de los costes).

El C-E que nos supondría introducir uno de estos programas frente a LI en el colectivo canino de las FFAA se ha realizado desde la perspectiva del sistema de salud pública actual.

Estos estudios son técnicas analíticas que comparan las consecuencias negativas (costes) y positivas (beneficios económicos y de salud) de los usos alternativos de recursos para extraer consecuencias sobre la eficiencia de estas intervenciones (Figura 2).

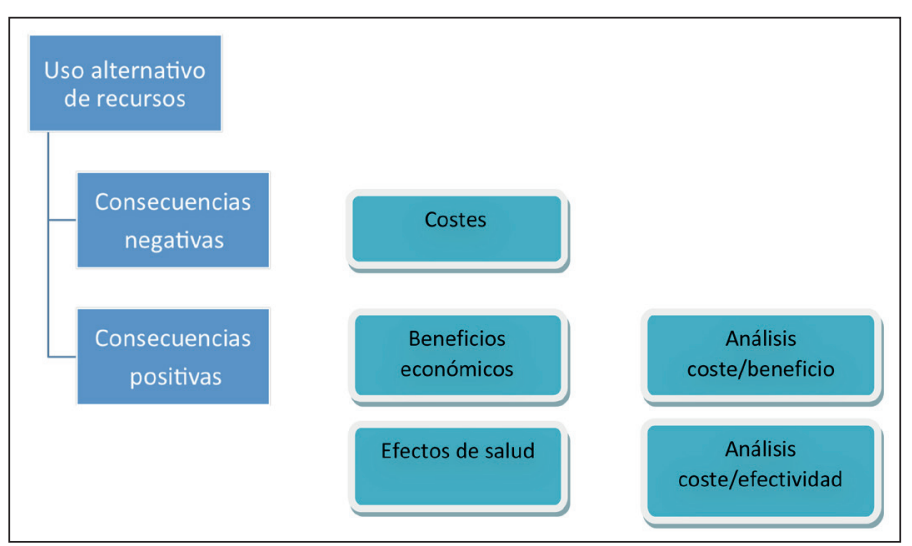

Figura 2. Diseño del estudio de eficiencia de un programa o de una intervención.

\section{Análisis coste-beneficio}

El análisis C-B se expresa en términos monetarios (Tabla 1).

Los dos parámetros fundamentales del análisis C-B son el valor actual neto, VAN, (net present value) y la razón beneficiocoste, $\mathrm{B} / \mathrm{C}$, (benefit cost-ratio):

$$
\mathrm{VAN}=(\mathrm{B}-\mathrm{C})_{\mathrm{t}} /(1+\mathrm{r})^{\mathrm{t}}=\mathrm{B}-\mathrm{C}
$$

$\mathrm{t}=$ número de años analizados en el horizonte temporal (entre $\mathrm{t}=0 \mathrm{y} \mathrm{N}$ )

$r=$ tasa de descuento en decimales. Los costes del programa y la efectividad se han actualizado para el año 2011 utilizando una tasa de descuento del 1\% (La tasa descuento para un análisis básico recomendada está entre el 1 y el 3\%).

$\mathrm{B}=$ Beneficios económicos programa de vacunación (coste de la enfermedad sin la vacunación -coste de la enfermedad con la vacunación)

$\mathrm{C}=$ Coste del programa de vacunación

$\mathrm{B}>\mathrm{C}$ : La intervención ahorra dinero

$\mathrm{B}=\mathrm{C}$ : Equilibrio costes-beneficios

$\mathrm{B}<\mathrm{C}$ : La intervención cuesta dinero (Coste Actual Neto).

El horizonte temporal viene definido por la duración de la protección (en nuestro caso, anual).

$$
\text { Razón } \mathrm{B} / \mathrm{C}=\sum \mathrm{B} / \sum \mathrm{C}
$$

$>1$ : Ahorra dinero

1: Equilibrio entre costes y beneficios

$<1$ Cuesta dinero

Cuando el análisis se realiza desde la perspectiva de la externalización, se incluyen tanto los costes directos como los indirectos. En cambio cuando el análisis se efectúa desde la perspectiva de la actuación en las FFAA solo se incluyen los costes directos. Se han incluido como costes indirectos los costes sociales.

Los costes de la enfermedad incluyen los gastos realizados para el diagnóstico y tratamiento de la enfermedad aguda y las complicaciones, así como los derivados de la pérdida de operatividad del PTM.

\begin{tabular}{|c|c|c|}
\hline Costes & FFAA & Externalización* \\
\hline Coste primovacunación CaniLeish ${ }^{\circledR}$ & $19,3 € /$ dosis & $45 € /$ dosis \\
\hline Coste Leisguard $^{\circledR} 60 \mathrm{ml}$ & $9,90 €$ & $12,47 €$ \\
\hline Administración de la vacuna & $0,90 € /$ dosis & Incluido en el coste de la vacuna \\
\hline Kit rápido de Inmmunodiagnóstico & $5,45 € /$ dosis & Incluido en el coste de la vacuna ${ }^{* *}$ \\
\hline Tto. efectos secundarios vacuna & - - & - - \\
\hline Envío por mensajería vacunas/provincia & $25 €$ & \\
\hline Compra de un Pastor Alemán para FFAA & $2.400 €$ & \\
\hline Pienso /anual & $528 €$ & \\
\hline Pruebas para alta en FFAA & $65,45 €$ & \\
\hline \multicolumn{3}{|l|}{ Diagnóstico de la enfermedad } \\
\hline . IFI & $18 €$ & $40 €$ \\
\hline - Analítica & $30 €$ & \\
\hline - Proteinograma & $22 €$ & \\
\hline \multicolumn{3}{|l|}{ Tratamiento de la enfermedad ${ }^{* * *}$} \\
\hline - Miltefosina & & $71,85 €$ \\
\hline - Alopurinol. & & $3,12 €$ \\
\hline - Metilglucamina antimoniato & & $12,47 €$ \\
\hline
\end{tabular}

Tabla 1. Desglose de los costes del estudio $C-B$ (en euros).

Fuente propia. ${ }^{*}$ Precio medio actualizado por consulta con diversas clínicas veterinarias de España. ${ }^{* *}$ No se incluye en el caso del medicamento. ${ }^{* * *}$ En los casos en los que se externalice dicho tratamiento. 
Análisis coste-beneficio y coste-efectividad, de las nuevas alternativas de inmunización surgidas en el mercado para la ...

El precio de compra de un PTM de raza pastor alemán es de $2.400 €$, el precio medio del pienso es de $44 € / 17 \mathrm{~kg}$, siendo el consumo de pienso diario de un perro atleta de raza de $500 \mathrm{~g}$ aproximadamente (dependiendo de la edad, estado físico, época del año, tipo de trabajo, y otras circunstancias, varia la dieta), por lo que el consumo en alimentación es de $528 € /$ año, a todo ello hay que sumarle los costes derivados de los trámites de alta de dicho PTM (30 €/radiografías para determinación de displasia de cadera y codo, y kits inmunodiagnóstico rápido y analítica completa).

El coste medio del tratamiento farmacológico se ha calculado a partir de la información sobre los tratamientos recomendados para la leishmaniosis canina de venta al público:

- Miltefosina ( $2 \mathrm{mg} / \mathrm{kg}$ oral SID durante 28 días)

- Metilglucamina antimoniato $(100 \mathrm{mg} / \mathrm{kg}$ SC SID durante 28 días).

- Alopurinol (10 mg/kg PO BID durante 7 meses).

El cálculo se ha realizado en relación al más empleado en la clínica para tratamientos agudos que es la miltefosina, y dependerá de la duración y la gravedad, variando el coste si suministramos uno solo o la combinación de ellos.

A los costes del tratamiento hay que añadirles los costes diagnósticos de la enfermedad: IFI, proteinograma y una analítica completa.

Los beneficios del programa de vacunación o de la intervención son los ahorros que se producen como consecuencia de la puesta en marcha de las acciones, es decir, la disminución en los costes de la enfermedad como consecuencia de la disminución de la incidencia de dicha enfermedad atribuible al programa de vacunación o a dicha intervención.

\section{Programa de vacunación}

Los costes de la primovacunación incluyen: los costes de la intervención así como los gastos derivados del tratamiento de las complicaciones postvacunales, que aunque raras las tendremos en cuenta.

Entre los costes directos sanitarios se han incluido costes veterinarios, coste de la vacuna, coste del kit diagnóstico rápido y costes no sanitarios como la remisión de la vacuna a las 20 provincias incluidas en los estudios de seroprevalencia de la enfermedad ${ }^{4}$.

El coste de las vacunas se ha calculado teniendo en cuenta que el precio de venta al Ministerio de Defensa por vacuna es de $19,3 € /$ dosis $(289,50 € / 15$ dosis). El coste de venta al público en una clínica veterinaria ronda los $45 € /$ dosis. No se ha evaluado el C-E de la estrategia de la revacunación anual, cuyo coste lo ciframos en $60 €$.

Los costes de administración de la vacuna $(0,90 €)$ incluyen: el material utilizado (algodón, alcohol) y costes de personal (25 $€ /$ visita), en el caso que por circunstancias, el servicio se prestase en una clínica privada.

El coste del tratamiento de los efectos postvacunales se ha calculado teniendo en cuenta que la vacunación puede producir efectos secundarios moderados (inflamación local leve y transitoria, con hipertermia transitoria ocasional) en uno de cada 100.000 animales vacunados, que requerirían 2 visitas a la clíni- ca, dependiendo de la gravedad y la duración del proceso, aunque por su escasa incidencia no lo incluiremos en el estudio.

\section{Nuevo medicamento preventivo.}

El coste del tratamiento preventivo con Leisguard ${ }^{\circledR} 60 \mathrm{ml}$, se ha calculado teniendo en cuenta que el precio de venta al Ministerio de Defensa sería de 9,90 €, y que en una clínica veterinaria su precio de venta al público es por término medio de 12,47€.

Por otro lado, tenemos que tener en cuenta dos datos importantes a la hora de realizar los cálculos; por un lado la dosificación empleada (para perros de más de $20 \mathrm{~kg}$ se necesitarían dos viales de $60 \mathrm{ml} / \mathrm{mes}$ ) y, por otro, el tratamiento a aplicar, que depende de la prevalencia de la enfermedad.

Los PTM que en la actualidad constituyen el grueso de nuestros efectivos (pastores alemanes) están clasificados como razas sensibles para contraer la enfermedad; es decir, están considerados como perros de alto riesgo, por esta circunstancia se deberían efectuar dos aplicaciones entre los meses de junio a octubre (época de mayor riesgo) y febrero a marzo (previo al test diagnóstico, realizado por el Servicio de Microbiología, Higiene y Sanidad Ambiental).

Nota: Hemos tenido presente las excepciones, ya que a los PTM que viajen de manera puntual a zonas endémicas como Líbano o Afganistán deberíamos de administrarles Leisguard ${ }^{\circledR}$ durante la estancia, y si el viaje es habitual adaptar el tratamiento preventivo según prevalencia y riesgo. Pero, este dato, no vamos a tenerlo en cuenta en el análisis matemático.

El coste del tratamiento de los efectos secundarios se han calculado teniendo en cuenta que la vacuna puede producir efectos secundarios debidos al comportamiento de la prolactina que puede afectar a la función adrenal (galactorrea y alterando otras funciones como la de la próstata, vesículas seminales y testículos) por lo que, como en el caso de los efectos secundarios de la vacuna, va a depender de la gravedad y la duración de estos y, aunque los tendremos en cuenta, no se incluyen en el estudio.

\section{Análisis coste-efectividad}

El análisis C/E se ha medido en términos de coste por año de vida ganado, en nuestro caso, mantenimiento de la operatividad de nuestro efectivo canino.

La efectividad, medida en términos de mantenimiento de operatividad del efectivo canino de las FFAA, se ha calculado a partir de la información sobre el número de animales referenciados positivos a leishmaniosis, obtenida de los estudios de prevalencia de dicha enfermedad, realizados por esta sección para el año 2010-2011.

Concluyendo: Teniendo una prevalencia del 2,25\%, la efectividad sería de $97,75 \%$.

\section{Programa de Vacunación}

Comparamos el coste neto del programa de vacunación (CaniLeish $^{\circledR}$ ) con su efectividad para la estrategia consistente en la 
vacunación de todo el efectivo canino considerando en principio la primovacunación (3 dosis/cada 3 semanas). No se considerará la inmunidad de grupo ni la pérdida progresiva de la eficacia de la vacunación a lo largo del tiempo debido a limitaciones en los datos disponibles.

La razón coste efectividad global se ha calculado utilizando la siguiente fórmula:

$\mathrm{C} / \mathrm{E}=\sum$ coste neto inicial $/$ efectividad $=\sum$ costes de primovacunación - reducción de costes clínicos veterinarios iniciales / $\sum$ efectividad.

El coste neto del programa de vacunación se ha calculado restando a los costes de vacunación la reducción de los costes de la leishmaniosis que se pueden conseguir mediante la vacunación en todo el efectivo canino de las FFAA, por lo que hay que tener en cuenta que a estos últimos se les debe aplicar el factor de reducción.

Los costes de vacunación incluyen las vacunas ( 3 dosis como primovacunación / individuo), los costes de administración de la vacuna y el coste del tratamiento de los efectos secundarios que puede producir la vacuna.

La reducción de los costes de la leishmaniosis asociada con la vacunación con CaniLeish ${ }^{\circledR}$ se ha calculado estimando, en primer lugar los costes clínicos veterinarios de la leishmaniosis canina en FFAA en el año 2011 y aplicando el factor de reducción.

Considerando que en el año 2011 ha habido 27 casos de leishmaniosis canina confirmados mediante IFI en el laboratorio del CEMILVETDEF, con bajo título de anticuerpos antileishmania, y leve sintomatología, y que no han necesitado atención hospitalaria e ingreso, entenderemos el coste clínico veterinario total como atención clínica primaria (número de animales atendidos, el tratamiento farmacológico recibido y pruebas diagnósticas) como:

$$
\text { El coste clínico veterinario total }=\sum_{\mathrm{t}=0}^{1} \text { Atención hospitala- }
$$
ria / $\mathrm{r}^{1}+\sum$ Atención clínica primaria $/ \mathrm{r}^{1}$

La vacunación puede reducir la enfermedad en un porcentaje que depende de la eficacia de la vacuna (E), de la cobertura de la vacuna (C) y el porcentaje de casos de LI causados por extractos del parásito incluidos en la vacuna $(\mathrm{V})$.

Este último factor puede excluirse debido a que se descarta el riesgo de infección inducida por la vacuna, pues como se explicó en la introducción. Una de las ventajas de CaniLeish ${ }^{\circledR}$ es que está compuesta por PSE y no por parásitos enteros, estimulando la inmunidad celular específica contra el parásito de LI por lo que no hay riesgo de recuperación de virulencia en ningún hospedador:

$$
\text { Factor de reducción }=\mathrm{C} \times \mathrm{E} \text {. }
$$

Este factor de reducción se ha calculado teniendo en cuenta una cobertura de la vacuna del 92,7 \% y una eficacia de la vacuna de $90 \%$ según los estudios realizados por los laboratorios Virbac. De acuerdo con estos datos el factor de reducción es de 83,43\%.

La eficacia de la vacunación en perros ya infectados no se ha investigado y por lo tanto no se puede recomendar. La inyección de la vacuna no demostró ningún beneficio en perros que habían desarrollado una leishmaniosis (infección activa o enfermedad) a pesar de la vacunación.

\section{Nuevo medicamento preventivo}

Comparamos el coste neto del nuevo medicamento preventivo (Leishguard ${ }^{\circledR}$ ) con su efectividad para la estrategia consistente en la implantación de su uso en nuestro efectivo canino.

La razón C-E global se ha calculado utilizando la siguiente fórmula:

$\mathrm{C} / \mathrm{E}=\sum$ coste neto inicial $/$ efectividad $=\sum$ costes de primovacunación - reducción de costes clínicos veterinarios iniciales / $\sum$ efectividad.

El coste neto del uso del medicamento preventivo se ha calculado restando a los costes del uso del medicamento, la reducción de los costes de la leishmaniosis que se pueden conseguir mediante su aplicación en todo el efectivo canino de las FFAA; hay que tener en cuenta que a estos últimos se le debe aplicar el factor de reducción.

El factor de reducción se ha calculado teniendo en cuenta una eficacia del medicamento del $80 \%$ y una cobertura del $90 \%$ según los estudios realizados por los laboratorios Esteve.

\section{RESULTADOS}

\section{Análisis C-B del programa de vacunación}

La eficiencia de los programas de vacunaciones no sistemáticas es variable, ya que la eficacia de las vacunaciones está muy influenciada por el coste de la vacuna y por la incidencia de la enfermedad (Tabla 2 y Figura 3).

\begin{tabular}{|c|c|c|}
\hline Costes & FFAA & Externalización* \\
\hline Coste de la vacunación(A) & 79.760 & 162.000 \\
\hline $\begin{array}{l}\text { Coste de la enfermedad Sin } \\
\text { Vacunación (B) }\end{array}$ & 85.328 & 84.788 \\
\hline $\begin{array}{l}\text { Coste de la enfermedad Con } \\
\text { Vacunación (C) }\end{array}$ & 642.065 & 651.725 \\
\hline $\begin{array}{l}\text { Beneficio de la vacunación. }(D)= \\
\text { (B)-(C) }\end{array}$ & -556.737 & -566.937 \\
\hline Valor Actual Neto. $(\mathrm{E})=(\mathrm{D})-(\mathrm{A})$ & -636.491 & -728.937 \\
\hline Razón Beneficio-coste. $(\mathrm{F})=(\mathrm{D}) /(\mathrm{A})$ & $\begin{array}{c}<0 \\
\text { NO AHORRA }\end{array}$ & $\begin{array}{c}<0 \\
\text { NO AHORRA }\end{array}$ \\
\hline
\end{tabular}

Tabla 2. Análisis C-B de la vacunación con CaniLeish ${ }^{\circledR}$ (en euros)

Fuente propia. ${ }^{*}$ Datos actualizados por consulta con diversa clínicas veterinarias de España.

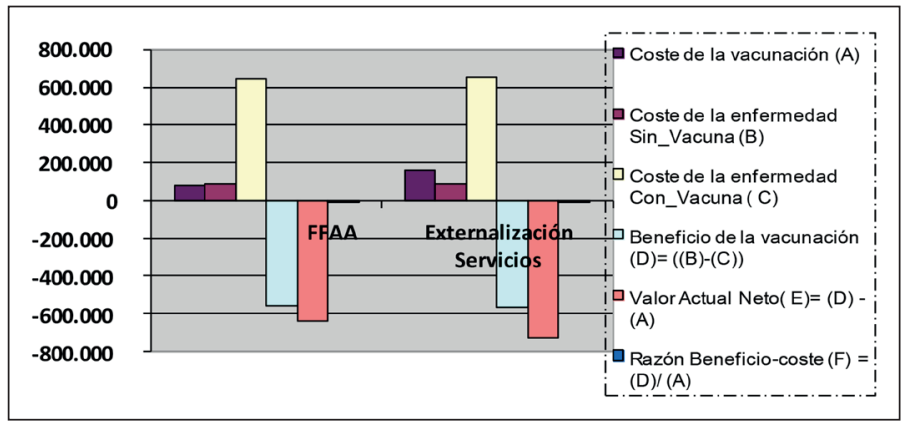

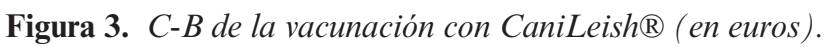


Análisis coste-beneficio y coste-efectividad, de las nuevas alternativas de inmunización surgidas en el mercado para la ...

El análisis C-B del uso medicamento preventivo Leisguard ${ }^{\circledR}$ se expone en la Tabla 3 y Figura 4.

Tabla 3. Análisis C-B del uso del medicamento preventivo (en euros).

\begin{tabular}{|c|c|c|}
\hline Costes & FFAA & Externalización* \\
\hline $\begin{array}{l}\text { Coste del uso del medicamento } \\
\text { preventivo(A) }\end{array}$ & 55.060 & 66.396 \\
\hline $\begin{array}{l}\text { Coste de la enfermedad Sin } \\
\text { Medicamento (B) }\end{array}$ & 85.324 & 84.788 \\
\hline $\begin{array}{l}\text { Coste de la enfermedad Con } \\
\text { Medicamento (C) }\end{array}$ & 769.412 & 766.879 \\
\hline $\begin{array}{l}\text { Beneficio del uso del } \\
\text { medicamento preventivo (D) = } \\
\text { (B)-(C) }\end{array}$ & -684.088 & -682.091 \\
\hline $\begin{array}{l}\text { Valor Actual Neto. ( E) = (D) } \\
\text { - (A) }\end{array}$ & -739.148 & -748.487 \\
\hline $\begin{array}{l}\text { Razón Beneficio-coste. }(\mathrm{F})= \\
\text { (D)/ (A) }\end{array}$ & $\begin{array}{c}<0 \\
\text { NO AHORRA }\end{array}$ & $\begin{array}{c}<0 \\
\text { NO AHORRA }\end{array}$ \\
\hline
\end{tabular}

Fuente propia. *Datos actualizados por consulta con diversa clínicas veterinarias de España.

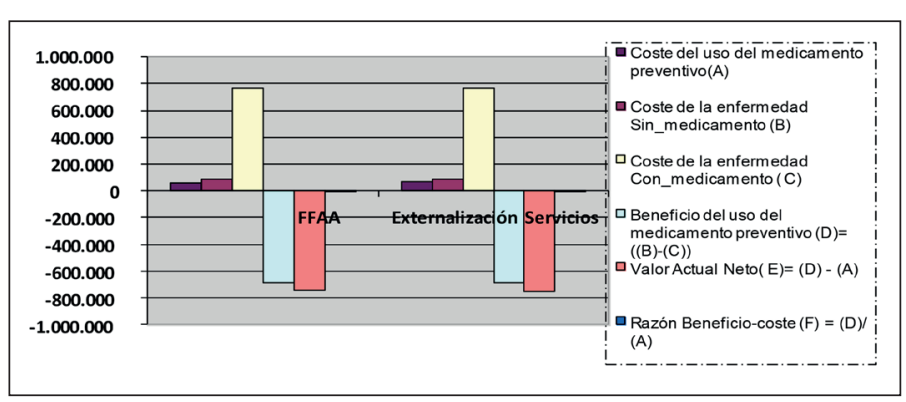

Figura 4. C-B del uso del medicamento preventivo (en euros).

Análisis C-E del programa de vacunación (Figura 5)

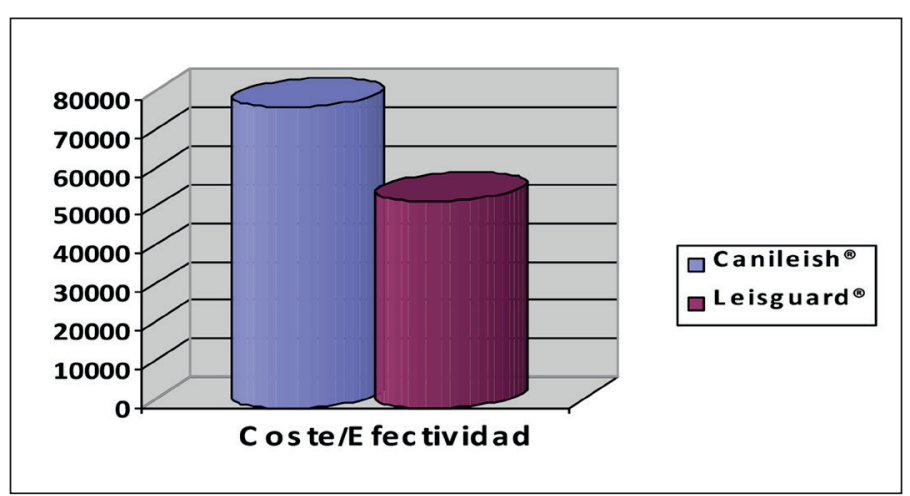

Figura 5. Análisis comparativo $C-E$ de ambos productos.

Las razones C-E global calculadas como se ha descrito en el apartado de Material y Métodos han sido:

- Razón $\mathrm{C} / \mathrm{E}=77.791 € /$ casos evitados con la primovacunación universal con CaniLeish ${ }^{\circledR}$.

- Razón $\mathrm{C} / \mathrm{E}=53.419 € /$ casos evitados con el uso del medicamento preventivo Leisguard ${ }^{\circledR}$

\section{DISCUSIÓN}

No debemos olvidarnos que, en el seno de las FFAA, nuestras actuaciones deben ir encaminadas hacia el control de los problemas de salud en las poblaciones (zoonosis) y la epidemiología es quien nos va a proporcionar la información necesaria para poder responder a las tres preguntas «hipocráticas de la prevención: La enfermedad, ¿es prevenible o controlable?, si la enfermedad es prevenible ¿cuáles son las estrategias de prevención y control más adecuadas? y, por último ¿cuál es la magnitud del beneficio de las estrategias de prevención?.

Hasta la fecha, el cumplimiento de la ITS 03/2007, nos deja un balance de 27 PTM afectados en el año 2011. Pese a que consideramos que es una ITS que diferencia de forma ambigua entre caso positivo y negativo a LI, por otra parte y a tenor de los resultados, entendemos que establece un procedimiento aceptable para el control y la prevención de la leishmaniosis.

No obstante, la aparición en el mercado (2012) de dos productos que son capaces de prevenir la enfermedad y que se nos pueden presentar como complemento al actual modelo de prevención y control que tenemos instaurados en las FFAA, nos ha movido a realizar este estudio.

Así mismo, para que el estudio tuviera más amplitud a la hora de poder tomar una decisión, los costes y beneficios de ambos modelos de inmunización (activa y pasiva) han sido comparados también con los costes que supondría la externalización de los servicios.

Estos estudios nos han permitido establecer un relación monetaria entre lo invertido y lo que estos programas o intervenciones revierten en ahorro por no tratar la enfermedad evitada y el ahorro a las FFAA que supone que los animales no padezcan la enfermedad (sobre todo en cuanto a la importancia del mantenimiento de la operatividad de nuestros PTM tanto en Territorio Nacional (TN) como en Zona de Operaciones (ZO).

En este estudio se imputan unos costes de la enfermedad y de la vacunación muy conservadores, es decir, en el caso de ausencia de datos de algún parámetro, se modelaba el peor de los supuestos (siempre en contra del modelo de inmunización aplicado), de esta forma, la utilización de los diferentes fármacos en el tratamiento de la enfermedad sería variable, por lo que hemos asumido lo que mayoritariamente se pautaría en caso de una leishmaniosis aguda.

En general, los costes globales de la vacunación y del tratamiento en ambos casos son superiores al coste del tratamiento de la enfermedad, con relaciones C-B negativas, es decir, no se produce ahorro de dinero. No obstante tiene su motivación realizar el análisis C-E para cuantificar el coste de prevenir la leishmaniosis, porque nos permite comparar entre programas o intervenciones en que la calidad de vida es un componente importante de resultado de salud.

Teniendo presente que la seroprevalencia anual de los PTM se ha mantenido desde el 2009 hasta la actualidad menor a la de los «perros civiles», concluimos que no es recomendable el desarrollo de la vacunación universal con Canileish ${ }^{\circledR}$, tanto por su C-E (77.791 €) por caso evitado, como por los costes de vacunación.

Si comparamos este resultado con el obtenido para el medicamento Leishguard ${ }^{\circledR}$ cuyo C-E sería menor por caso evitado 
(53.419€), por esto la estrategia de administrar el medicamento, supondría un incremento de costes menor, por lo que desde el punto de vista económico sería la más «adecuada».

Pese a que podríamos intentar actuar como «cliente único», aprovechando nuestro enorme potencial (más de 1.000 PTM) para que se nos llegara a considerar frente a terceros, como cliente preferencial y obtener así economías de escala, consideramos que no lograríamos un beneficio frente a las actuaciones que estamos realizando hasta la fecha; y esto lo justificamos porque hemos actuado como un «cliente inteligente» (constante preparación y estudio) para informarnos y conocer en cada momento el avance biotecnológico (las nuevas terapias) y con ello, poder efectuar una correcta evaluación de costes y ofrecer el mejor asesoramiento al Mando.

\section{CONCLUSIONES}

A tenor de los datos obtenidos en nuestro estudio concluimos que:

1. No es recomendable el desarrollo de la vacunación universal.

2. No es recomendable la administración del medicamento preventivo.

3. Cualquier medida preventiva nueva (que en este caso, ha quedado demostrado que supondría un incremento de costes menor que el de la vacunación), debe considerarse siempre a medio/corto plazo, y siempre en función de los cambios epidemiológicos de la enfermedad en cuestión.

4. Proponemos recurrir a la epidemiología de campo como herramienta imprescindible de trabajo para poder alcanzar nuestro objetivo sanitario estratégico: la vigilancia epidemiológica y la inteligencia sanitaria.

5. Debemos efectuar siempre los pertinentes estudios epidemiológicos y económicos, con el objetivo de asesorar al Mando y que este gestione directamente la logística no operativa, teniendo siempre todos los datos actualizados por un equipo multidisciplinar.

6. Racionalizar el proceso logístico de la adquisición y apoyo durante el ciclo de vida de los PTM.

7. Revisar periódicamente las ITS.

\section{BIBLIOGRAFÍA}

1. Cordero del Campillo M, Rojo Vázquez FA. Parasitología Veterinaria. McGraw-Hill Interamericana. Madrid, 1999:652-665.

2. Encinas Aragón J, Fernández Gómez FJ, Lasheras Carbajo MD, Barbas del Buey FJ. Leishmaniosis canina y humana. Una visión de conjunto. Revista Profesión Veterinaria. Colegio Oficial de Veterinarios de Madrid. (consultado el 10/05/2012]. Disponible en URL: http://www.colvema.org/pdf/leishmaniosis.pdf

3. Instrucción Técnica Sanitaria 03/2007 «Control de Leishmaniosis y Filariosis en los efectivos caninos de las Fuerzas Armadas», de la Subsecretaria de Defensa, Inspección General de Sanidad.

4. Estudio seroepidemiológico de las zoonosis especificadas en la ITS 03/2007. Trabajo realizado por la Sección de Análisis Clínicos, Parasitología y Epi- demiología del Servicio de Microbiología, Higiene y Sanidad Ambiental del CEMILVETDEF. Abril 2009.

5. Baly Gil A, Toledo MA, Rodríguez Jústiz F. La economía de la salud, la eficiencia y el costo de oportunidad. Rev Cubana Med Gen Integr 2001; 17(4):395-398.

6. Laboratorios Virbac España. CANILEISH. (consultado el 10/05/2012). Disponible en URL: http://www.virbac.es/p-virbacespubes/display. $\underline{\text { aspx }}$ ?srv $=p$-virbaces $\&$ typ $=$ pub\&lang $=e s \& \mathrm{cmd}=$ view \&style $=$ styles $/$ specie. xsl\&select=PRODUCT[@ID\$eq\$PRODUCT 141]\&

7. Rosa R, Rodríguez OR, Marques C, Santos-Gomes GM. Leishmania infantum: soluble proteins released by the parasite exert differential effects on host immune response. Experimental parasitology. 2005; 109:106-114.

8. Laboratorios Esteve. ESTEVE Veterinaria lanza LEISGUARD ${ }^{\circledR}$, el primer fármaco que previene y trata la leishmaniosis canina. (Consultado 10/5/2012). Disponible en URL: http://www.esteve.es/EsteveFront/PressRoom.do?op $=\mathrm{DN} \& \operatorname{div}=$ vet $\&$ con $=3347$

9. Llinás J, Gómez-Ochoa P, Sabaté D, Homedes J, Ferrer L. Clinical efficacy of a domperidone-based treatment program for the prevention of canine leishmaniosis. Proceedings of the 46 th AVEPA-SEVC Congress.2011. (Consultado el 15/05/2012). Disponible en URL: http://www.esteve.es/EsteveArchivos/ consultasonline/leispro/en/46th AVEPA SEVC congress 2011.pdf

10. Barone JA. Domperidone: A Peripherally Acting Dopamine2-Receptor Antagonist. Ann Pharmacother.1999; 33(4):429-440.

11. Hall JA, Washabau RJ. Gastric prokinetic agents. In: Bonagura J.: Kirk's Current Veterinay Therapy XIII Small Animal Practice.2000; 614-617.

12. Kato H, Fujino T, Aramaki S, Koresawa M, Yamashita S, Torigoe T. The role of Domperidone in the regulation of prolactin rease in rats. Life Sciences. 1980; 26 (16), 1343-1347

13. Fujino T, Kato H, Yamashita S, Aramaki S, Morioka H, Koresawa M. et al. Effects of Domperidone on serum prolactin levels in human beings. Endocrinol Jpn.1980; 27(4):521-525.

14. Matera L. Action of pituitary and lymphocyte prolactin. Neuroimmunomodulation. 1997; 4(4):171-180.

15. Matera L, Mori M. Cooperation of Pituitary Hormone Prolactin with Interleukin- 2 and Interleukin-12 on Production of Interferon-Y by Natural Killer and T Cells. Annals New York Academy of Sciences.2000; 505-513.

16. Matera L, Mori M, Galetto A. Effect of prolactin on the antigen presenting function of monocyte-derived dendritic cells. Lupus. 2001; 10(10):728-734.

17. Płociński P, Dzitko K, Długońska H. Prolactin as a modulator of antiparasitic immunity. Wiad Parazytol. 2007; 53(4):263-270.

18. Gómez-Ochoa P, Sabaté D, Homedes J, Ferrer L. Use of the nitroblue tetrazolium reduction test for the evaluation of Domperidone effects on the neutrophilic function of healthy dogs. Vet Immunol Immunopathol. 2012; 146(1):97-99. (Consultado el 15/5/2012). Disponible en URL: http://www. esteve.es/EsteveArchivos/consultasonline/leispro/en/vet immunol immunopathol 2012.pdf

19. Gómez-Ochoa P, Castillo JA, Gascón M, Zarate JJ, Alvarez F, Couto CG. Use of domperidone in the treatment of canine visceral leishmaniasis: a clinical trial. Vet J.2009; 179(2):259-263. (Consultado el 15/05/2012). Disponible en URL: http://www.esteve.es/EsteveArchivos/consultasonline/leispro/en/ the veterinary_journal_179_2009.pdf

20. Paltrinieri S, Solano-Gallego L, Fondati A, Lubas G, Gradoni L, Castagnaro $\mathrm{M}$, et al. Guidelines for diagnosis and clinical classification of leishmaniasis in dogs. J Am Vet Med Assoc. 2010; 236(11):1184-1191.

21. López Bastida J, Oliva J, Antoñanzas F, García-Altés A, Gisbert R, Mar J, et al. Propuesta de guía para la evaluación económica aplicada a las tecnologías sanitarias. Gaceta Sanitaria. 2010; 24(2):154-170.

22. Shepard DS. Cost-effectiveness in Health and Medicine. By M.R. Gold, J.E Siegel, L.B. Russell, and M.C. Weinstein (eds). New York: Oxford University Press, 1996. The Journal of Mental Health Policy and Economics. 1999;2(2):91-92.

23. Salleras L, Domínguez A. Evaluación de la eficacia de las vacunas y de la efectividad de los programas de vacunaciones. En: Salleras L, editor. Vacunaciones preventivas. Principios y aplicaciones. Barcelona, 2003: 781-799. 